ventionsempfehlungen für Erwachsene, die AU-Bescheinigungen und die Dokumentation der DMP COPD.

Entlastungen von mehr als 100.000 Stunden gab es bei der Aufklärung der Patienten bei Überschreiten der Festbetragsgrenze, bei der Bescheinigung für dauerbehandelte Krankheit, bei der Erhebung von Daten im Ersatzverfahren, beim Antrag auf Fortsetzung psychiatrischer Behandlungen, beim Antrag Kurzzeittherapie sowie beim Ausstellen von Überweisungen. Fortschritte gebe es bei der Vereinheitlichung und Vereinfachung von Formularen. So ließen sich Laborüberweisungen zum Beispiel jetzt papierlos erledigen.

Aufgenommen haben die Wissenschaftler Vorgaben des G-BA, aus dem Bundesmantelvertrag, der BÄK sowie aus Vereinbarungen der KBV mit der Unfallversicherung, der Polizei und der Bundeswehr. Nicht enthalten sind zum Beispiel Vorgaben der PKV.

\section{Projekt "Mehr Zeit für die} Behandlung"

Auch der Nationale Normenkontrollrat beschäftigt sich seit einiger Zeit mit dem Thema Bürokratieabbau in Arztpraxen, mit etwas positiverer Attitüde als die KBV. „Mehr Zeit für Behandlung“ lautet der Name des Projekts. Ratsmitglied und Projektleiter Wolf-Michael Catenhusen zog im Oktober ein positives $\mathrm{Fa}$ zit. Von den im August 2015 vereinbarten 20 Handlungsempfehlungen seien rund die Hälfte vollständig umgesetzt. 13 Handlungsempfehlungen betreffen die Vertragsärzte und -psychotherapeuten. Etwa zehn seien umgesetzt oder kurz vor dem Abschluss. Konkret handelt es sich dabei um Erleichterungen bei der Verschreibung von Arbeitsunfähigkeit, das vereinfachte Antragsverfahren bei der Psychotherapie, die Umsetzung einrichtungsinternen Qualitätsmanagements, die Chronikerbescheinigung (Muster 55) und die Verordnung von Re- habilitationsleistungen. Die Blankoformularbedruckung erspart Ärzten Formulare vorrätig zu halten. Elektronische Ausfüllhilfen und das einrichtungsinterne statt arztbezogene Qualitätsmanagement erleichtern ebenfalls das Leben.

Noch unterwegs ist die Verschlankung der Anfragen von Krankenkassen und Medizinischen Diensten. Das gilt ausweislich der Dokumentation des Normenkontrollrats auch für Verordnung von Heilmitteln. Als fortgeschritten sieht der Rat die Beratungen zur Verordnung von Krankentransporten an. Noch keine Ergebnisse gibt es zum Beispiel bei der Verschreibung von Betäubungsmitteln oder der Bescheinigung von Arbeitsunfähigkeit während der Wiedereingliederung.

Scharfe Kritik äußerte Catenhusen an der Haltung der Krankenhäuser und der Länder. Die Weigerung der Länder, den Bürokratieabbau mitzutragen, belaste den Prozess.

Anno Fricke

\title{
Existenzgründung als sichere Bank
}

Für Existenzgründer hat die aktuelle Niedrigzinsphase auch ihre guten Seiten: Zurzeit entfallen im Durchschnitt nur 1 bis $2 \%$ der Praxiskosten auf die Zinsbelastung durch die Praxisfinanzierung. Das schafft Spielraum beispielsweise für die Personalkosten.

$\mathrm{D}$ as finanzielle Risiko der Niederlassung ist eigentlich sehr gering“, ermutigte Dominik Hanisch von der Deutschen Apotheker- und Ärztebank seine Zuhörer bei einem Workshop zum Thema „Finanzierung einer Privatpraxis optimal gestalten“ auf dem Tag der Privatmedizin in Frankfurt am Main. Es liege gerade bei 0,05\% der Finanzierungen der Bank. Wenn es überhaupt einmal zu einer Insolvenz komme, dann sei das in der Regel nicht auf die Praxis zurückzuführen, sondern liege eher an privaten Problemen, führte der Filialleiter der Standesbank in Darmstadt weiter aus.

Als Orientierungsrahmen für die Personalkosten als wichtigsten Kostenblock nannte Hanisch beispielsweise für Allgemeinärzte etwa $26 \%$ der Gesamtkosten. „Das sollten sie auch ausgeben“, empfahl er. Denn in vielen Praxen sei mittlerwei- le fehlendes gutes Personal eine echte Bremse für die Weiterentwicklung. Der Fachkräftemangel mache sich auch in Arztpraxen längst bemerkbar.

Die Raumkosten lägen im Schnitt bei 6 bis $7 \%$ und seien damit ebenfalls ein wichtiger Faktor. Bei der Niederlassung in einer übernommenen Praxis sei es wichtig, darauf zu achten, wie lang der Mietvertrag noch laufe - aber auch, ob gegebenenfalls Möglichkeiten einer Vergrößerung bestehen. Die Praxisbewertung ist bei einer Privatpraxis erfahrungsgemäß nicht leicht, weil die Patientenbindung an den abgebenden Arzt häufig besonders eng ist. Als Faustformel für einen Preis nannte Hanisch einen nachhaltigen Jahresgewinn als Minimum plus Abschreibungen plus den materiellen Wert obendrauf. Aber die Bank halte sich bei der individuellen Preisbildung heraus, könne jedoch un- terstützend zur Kaufpreisfindung eine Beratung anbieten. Hier spielten viele Faktoren hinein, etwa die Konkurrenzsituation, die Entwicklung von Fallwert und Fallzahl, die Altersstruktur der Patienten.

Bei großen Investitionen wie einer Existenzgründung sei es zudem wichtig, vorab durchzurechnen: „Welchen Umsatz muss ich haben, damit die Praxis funktioniert und mein eigener Geldbedarf gedeckt ist?" Nur wenn das realistisch erscheine, sei die Existenzgründung sinnvoll. Bei Finanzierungen sollten die Ärzte außerdem beachten, dass sie kurzfristigen Bedarf, etwa eine kurze Unterdeckung im Konto, auch kurzfristig finanzieren, also zum Beispiel mit dem Kontokorrent. Investitionen in langlebige Geräte könnten dagegen mit lange laufenden Darlehen bezahlt werden.

Nicht zuletzt empfahl Hanisch für einen erfolgreichen Start, dass sich der Existenzgründer bereits vor der Niederlassung überlege, was das Alleinstellungsmerkmal der Praxis sein könnte, wen die Praxis besonders als mögliche Patienten ansprechen und wie das Praxisteam Patienten begegnen will.

Hauke Gerlof 